Anaesthesist 2018 $67: 321-325$ https://doi.org/10.1007/s00101-018-0444-3 Online publiziert: 7. Mai 2018

(c) Springer Medizin Verlag GmbH, ein Teil von Springer Nature 2018

CrossMark

\title{
S. Eifert
}

Herzchirurgische Klinik und Poliklinik, Transplantationszentrum, Klinikum Großhadern, LMU München, München, Deutschland

\section{Perkutane und chirurgische Optionen der mechanischen Kreislaufunterstützung in der Therapie der terminalen Herzinsuffizienz}

sind multifaktoriell. Die medikamentöse Therapie der fortgeschrittenen Herzinsuffizienz hat eine enorme Entwicklung genommen; sie ist als hocheffizient $\mathrm{zu}$ betrachten, besonders mit der Einführung der Angiotensinrezeptorblocker sowie des Präparats Entresto ${ }^{\circledR}$ (Sacubitril; Neprilysininhibitor in Kombination mit Valsartan) als auch des Kalzium-Sensitizers Levosimendan. Dies ist mit einer Stabilisierung des Krankheitsstatus, einer Verlangsamung der Progression sowie der Zunahme der durchschnittlichen Lebenserwartung verbunden. Diese Erklärung wird auch durch Strüber et al. [2] unterstützt: Bereits seit der Jahrtausendwende führte die OMT zunächst zu einer Abnahme der Meldungen für eine Herztransplantation. Jedoch nach Ausschöpfung dieser Optionen wurde die Transplantation zu einem späteren Zeitpunkt angestrebt. Im Jahr 2001 wurde das Allokationsverfahren verändert: Transplantate sollten in erster Linie hochdringlichen Empfängern zugeordnet werden. Eine weitere Erklärung ist die allgemeine demografische Entwicklung in unserem Land mit der Zunahme der Inzidenz von terminaler Herzerkrankung.

Gesamteuropäisch betrachtet, können nur $25 \%$ Patienten, die zur Überbrückung ein Herzunterstützungssystem erhalten, innerhalb eines Jahres nach Implantation transplantiert werden. Somit entsteht zwangsläufig der Status einer chronischen Kreislaufunterstüt- zung durch vorrangig linksventrikuläre Unterstützungssysteme („left ventricular assist device“, LVAD) bei Patienten, die ambulant betreut werden können und auf unbestimmte Zeit mit einem solchen Gerät leben müssen.

\section{Technische Entwicklung von Kunstherzsystemen}

Vor knapp 50 Jahren stand das Thema Herzersatz bereits im Mittelpunkt des öffentlichen Interesses; zum einen wegen der ersten Herztransplantation im Dezember 1967, zum anderen, weil damals die Entwicklung und der Einsatz von Kunstherzsystemen begannen. Dies bedeutete zunächst den vollständigen Herzersatz durch ein pneumatisch angetriebenes Zweikammeraggregat. Damals wies das Kunstherz erhebliche technische Mängel auf, und der Antrieb über riesige extrakorporale Konsolen machte eine permanente Therapie nicht praktikabel.

Um technischen Problemen zu begegnen, verlegte man zunächst die künstlichen Ventrikel nach außen. Aus dem Herzersatz war somit ein Herzunterstützungssystem entwickelt geworden. Diese Technik konnte erfolgreich eingesetzt werden und wird bis heute angeboten. Die Entwicklung vom biventrikulären Herzersatz zur isolierten linksventrikulären Unterstützung nahm ihren Lauf. In den 1990er-Jahren wurden implantierbare, elektrisch angetriebene, linksvenläufig. Die Ursachen dieser Entwicklung 


\begin{tabular}{|c|c|c|c|c|}
\hline $\begin{array}{l}\text { INTERMACS } \\
\text { Score }\end{array}$ & NYHA & $\begin{array}{l}\mathrm{ACCl} \\
\mathrm{AHA}\end{array}$ & Zustand des Patienten & \\
\hline 1 & IV & D & Kritischer kardiogener Schock & Stunden \\
\hline 2 & IV & D & Steigender Katecholaminbedarf & Tage \\
\hline 3 & IV & D & Stabil unter Inotropika & Woche \\
\hline 4 & IV & D & Häufige Dekompensation & Woche- \\
\hline 5 & IV & C & Ruhebeschwerden/nicht belastbar & \\
\hline 6 & IV & C & Ruhebeschwerden/kaum belastbar & Variabel \\
\hline 7 & Illb & C & Kaum belastbar & $\begin{array}{l}\text { 1-JÜR } \\
50-70 \%\end{array}$ \\
\hline
\end{tabular}

Abb. 1 A Klassifikation der terminalen Herzinsuffizienzanhand der INTERMACS-Einteilung in Relation zu NYHA- und ACC/AHA-Klassifikation. INTERMACS Interagency Registry for Mechanically Assisted Circulatory Support, NYHA New York Heart Association, ACC Association Canadian Cardiology, AHA American Heart Association, JÜR Einjahres-Überlebensrate

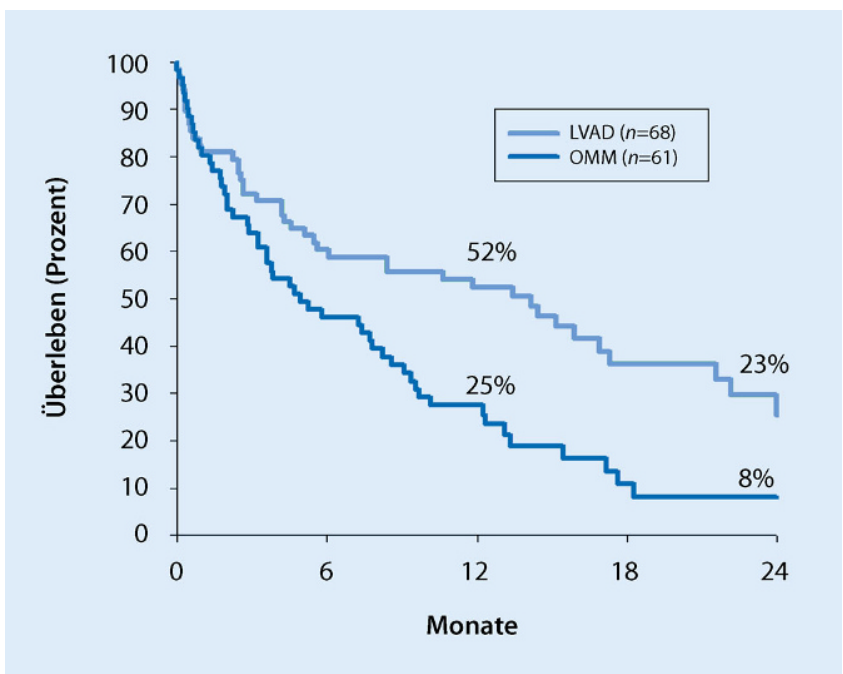

Abb. $2 \triangleleft$ REMATCH-Studie. OMM: "optimized medical therapy", LVAD: „left ventricularassist device"

trikuläre Unterstützungssysteme produziert. Diese großen und schweren Verdrängerpumpen wurden in eine subdiaphragmale Tasche implantiert. Zum elektrischen Betrieb wurde ein perkutanes Kabel transkutan nach außen geführt: So entstand die auch noch heute am häufigsten verwendete Konfiguration. Die Größe und der Geräuschpegel der Geräte, die Infektionsgefahr sowie die mangelhafte mechanische Haltbarkeit stellten Limitationen dar [2, 3].

Die Technik der zweiten und dritten Generation von LVAD hat jedoch heute neue Möglichkeiten eröffnet: Diese LVAD arbeiten klappenfrei mit kontinuierlichem Blutfluss. Die neueren Generationen von Systemen werden elektrisch angetrieben und erzeugen über einen Ro- tor einen kontinuierlichen, nichtpulsatilen Blutfluss: die so genannten Axialbzw. Zentrifugalpumpen, die erheblich kleiner und geräuschlos sind. Darüber hinaus sind sie mechanisch über Jahre stabil. Der wesentliche Unterschied zwischen einer Axial- und Zentrifugalpumpe liegt im Design des Rotors. Daraus resultieren unterschiedliche Eigenschaften zur Modulation der Vor- und der Nachlast. Vorteile der Zentrifugalpumpe sind die geringe Hämolyserate und geringe Thrombogenität, einfache Handhabung und geringe Störanfälligkeit $[4,5]$.

Es stellte sich heraus, dass der kontinuierliche Blutfluss und die Pulslosigkeit physiologisch unproblematisch sind. Die mit der schweren Herzinsuffizienz verbundenen neurokognitiven Störungen konnten mit pulslosen LVAD in gleicher Weise wie mit pulsatilen Geräten verbessert werden. Der Patient ist darüber hinaus in der Lage, eine zunehmende körperliche Belastung durch sein entlastetes natives Herz zu kompensieren.

Wie von Strüber et al. gezeigt wurde, ist die Inzidenz von Infektionen und neurologischen Störungen - auch in Abhängigkeit von der operativen Technik rückläufig: So wurden noch in der REMATCH-Studie für ein LVAD der ersten Generation schwere neurologische Störungen mit einer Inzidenz von 0,39/ Patientenjahr beschrieben, in einer Untersuchung mit einer Axialpumpe (zweite Generation) waren es noch 0,18 Schlaganfälle/Patientenjahr, und in einer Studie mit einem Gerät der dritten Generation wurde ebenfalls eine Inzidenz von 0,18 genannt $[1,2]$.

Bei implantierbaren elektrisch betriebenen pulsatilen Systemen wird die benötigte Energie über ein Verbindungskabel oder per Induktion zwischen 2 Spulen über die unverletzte Haut von außen nach innen transportiert.

In ihrem Artikel „Minimalinvasive und operative Optionen zur mechanischen Kreislaufunterstützung in der Herzinsuffizienz" geben die Autoren einen umfassenden Überblick über die wichtigsten aktuellen Verfahren zur mechanischen Kreislaufunterstützung. Die Autoren kategorisieren die verfügbaren Methoden und teilen die mechanische Kreislaufunterstützung nach Zugangsart sowie nach Dauer ihrer Anwendung in Systeme für kurzfristige sowie für mittelbis langfristige Anwendung ein. Derwall et al. [6] stellen die unterschiedlichen verfügbaren, zugelassenen Systeme pulsatiler und kontinuierlicher Zirkulation vor. Sie unterscheiden intra- von extrakorporalen Pumpen.

\section{Perkutane Unterstützungs- systeme}

Die minimalinvasiv applizierbaren linksund rechtsventrikulären Unterstützungssysteme stellen zunehmend die zum Einsatz kommende Variante des kurzzeitigen mechanischen Kreislaufersatzes dar. Vor- und Nachteile der aufgeführten Systeme werden durch die Autoren aufge- 


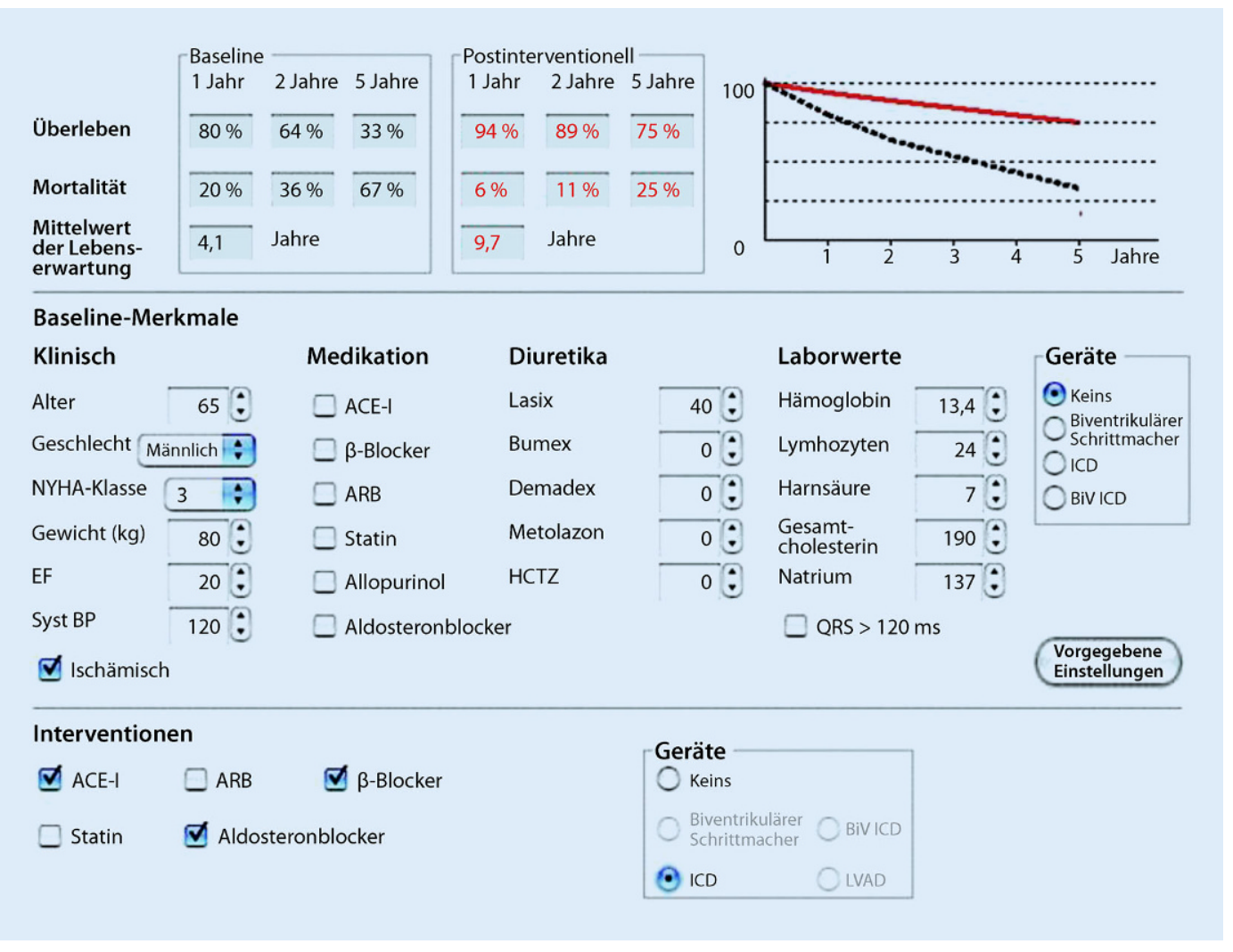

Abb. $3<$ Seattle Heart Failure Model. NYHA New York Heart Association, EF Ejektionsfraktion, Syst $B P$ systolischer Blutdruck, ACE-I Angiotensinkonversionsenzym-Inhibitor, $A R B$ Angiotensinrezeptorblocker, HCTZ Hydrochlorothiazid, QRS QRS-Dauer, ICD implantierbarer Kardioverter-Defibrillator, BiV ICD biventrikulärer ICD, LVAD „left ventricular assist device“ zeigt; besonders der Vorteil der geringeren Invasivität bei den perkutanen, katheterbasierten Verfahren wird detailliert geschildert.

Begonnen wird mit der intraaortalen Ballonpumpe, dem am längsten klinisch verfügbaren perkutanen System. Die Implantationstechnik wird detailliert beschrieben.

\section{Extrakorporaler Life-Support (ECLS)/extrakorporale Membran- oxygenierung (ECMO)}

Eine kurzzeitige Unterstützung der kardialen Funktion kann mittels venoarterieller ECMO (Nomenklatur ECLS) bis 14 Tage erfolgen; im Unterschied dazu wird die venovenöse ECMO für pulmonales Versagen verwendet. Indikationen zur ECLS-Anlage bestehen im Links- und/oder Rechtsherzversagen, als „bridge to transplant“, bei Risikointerventionen, für den kathetergestützten transfemoralen Aortenklappenersatz (TAVI), im Rahmen der Postkardiotomie und unter Reanimation. Sowohl die perkutane als auch die offene operative Implantationstechnik finden Anwen- dung. Derwall et al. erläutern umfassend die wesentlichen Kriterien, inklusive positiver und negativer Effekte, dieser Form der mechanischen Kreislaufunterstützung: die Fähigkeit zur Perfusatmodifikation und die Möglichkeit der Nutzung zur Absorption einerseits und die Unmöglichkeit zur linksventrikulären Entlastung (fehlendes „unloading“) bei gleichzeitiger Erhöhung der linksventrikulären Nachlast andererseits.

\section{Operative Therapie}

Erst im Endstadium werden Patienten einer operativ-chirurgischen Therapie zugeführt und bislang nur selten frühzeitig in der interdisziplinären oder herzchirurgischen Herzinsuffizienzambulanz vorgestellt.

\section{Zugang}

Der konventionelle Zugang erfolgt über eine mediane Sternotomie, v.a., wenn weitere chirurgische Eingriffe nötig sind oder es sich um eine Reoperation handelt. Dieser Eingriff wird mithilfe der extrakorporalen Zirkulation durchgeführt.
Eine Kardioplegie ist - je nach Ausmaß des Eingriffs - nicht zwingend erforderlich. Der Einflusstrakt des Unterstützungssystems wird über einen Haltering am Herzen befestigt und mittels Gefäßgraft an die Aorta ascendens anastomosiert. Das Kabel, das das Unterstützungssystem mit dem sog. Kontroller verbindet, wird über den Bauchraum ausgeleitet. Anschließend wird die Funktion vorrangig des linken Ventrikels schrittweise durch das Unterstützungssystem übernommen, bis eine vollständige Funktionsübernahme etabliert ist.

Alternativ kann die Operation am schlagenden Herzen ohne EKZ über eine linksseitige anterolaterale Ministernotomie stattfinden. Diese Option ist bei später geplanter Herztransplantation eine gute Vorgehensweise. Für die Anastomosierung des aortalen Grafts kommen die Aortae ascendens et descendens infrage (obere Teilsternotomie oder eine parasternale Inzision rechts). Der Anschluss über die Aorta descendens (über laterale Thorakotomie) ist technisch aufwendiger und bietet z.T. schlechtere Sichtbarkeit für den Chirurgen. Alternativ stellt die linke A. subclavia 


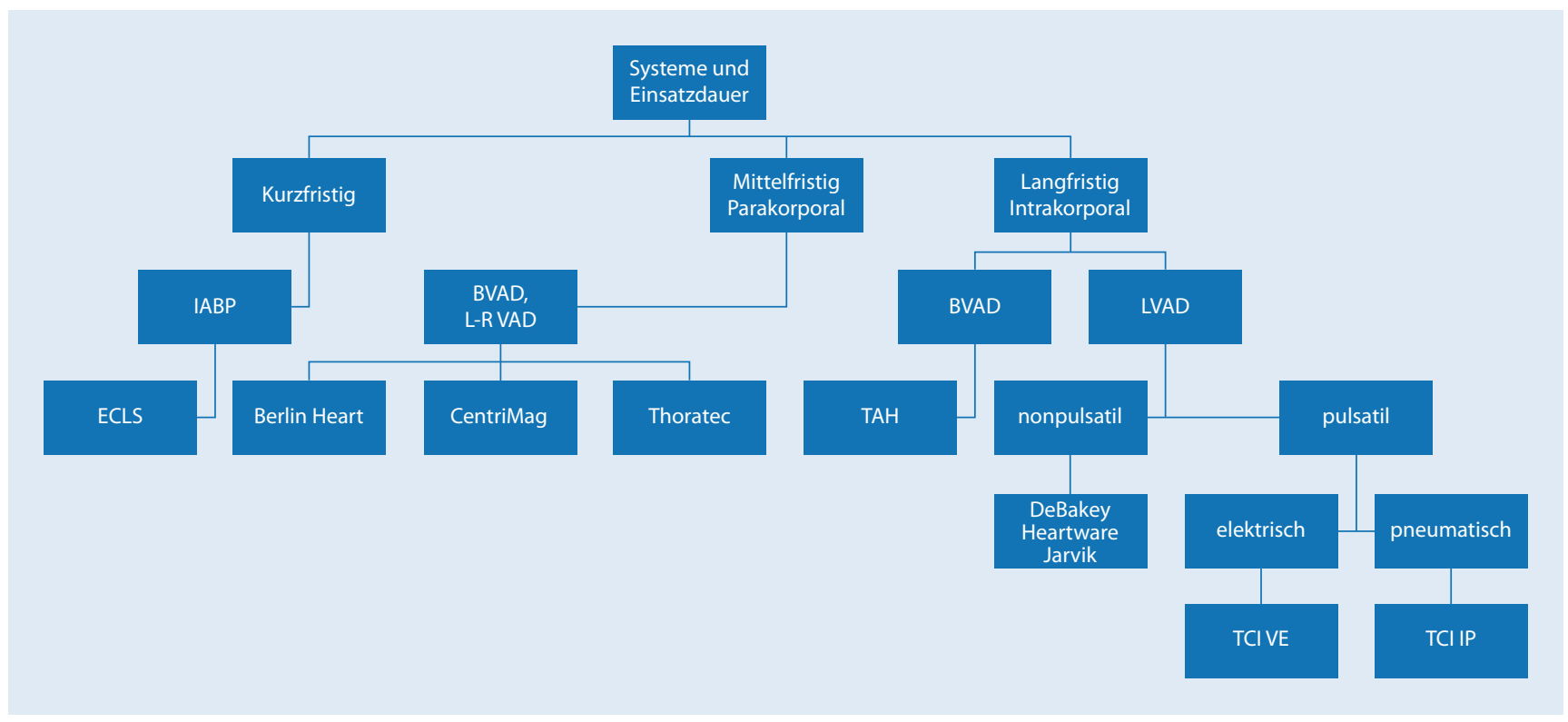

Abb. 4 ム Übersicht über verfügbare „assist devices"

eine Option dar (subklavikuläre Inzision links). Die Auswahl der Anschlussgefäße richtet sich nach der individuellen Anatomie des Patienten.

\section{Ventrikuläre Unterstützungs- systeme}

Die Indikationsstellung wird von Derwall et al. klar geschildert. Hinzu kommt, dass aufgrund des jeweils aktuellen Status des Patienten nicht nur hämodynamische Parameter relevant sind, sondern Indikatoren für eine beginnende Organdysfunktion. Diese sind über verschiedene Scoring-Systeme berechenbar und ermitteln nicht nur das Risiko des operativen Eingriffs, sondern auch den Erfolg der Operation im Sinne des Überlebens. Das Seattle Heart Failure Model (SHFM; - Abb. 3; [7]) wurde als interaktives Programm implementiert und wendet den Seattle Heart Failure Score an. Er schätzt die mittlere, die Ein-, Zwei- und Fünfjahresüberlebensrate und die Erfolgschancen der medikamentösen als auch der implantierten Geräte für den individuellen Patienten ab, falls die Indikation gegeben ist. Darüber hinaus kann zur Beurteilung der Funktion des rechten Herzens, v.a. zur präoperativen Abschätzung der kürzlich entwickelte EUROMACS (European Registry for Patients with Mechanical Circulatory Support) Right-Sided
Heart Failure Risk Score herangezogen werden [8].

Bei der operativen Behandlung der terminalen Herzinsuffizienz steht eine Vielzahl an Geräten zur links- (LVAD), rechts- („right ventricular assist device“, RVAD) und biventrikulären („biventricular assist device“, BiVAD) Unterstützung zur Auswahl (• Abb.4).

In $97 \%$ der Fälle findet weltweit die Implantation eines LVAD statt $[9,10]$.

Patienten, die eine Indikation zur Implantation eines LVAD aufweisen, zeigen ohne chirurgische Therapie (unter maximaler medikamentöser Behandlung) eine Einjahresüberlebensrate von $10-15 \%$. Damit übersteigt dieser Prozentsatz bei der schweren Herzinsuffizienz im NYHA-Stadium IV die Sterblichkeit maligner Erkrankungen deutlich und stellt die dritthäufigste Todesursache in Deutschland dar. Die Einjahresüberlebensrate nach LVADImplantation liegt hingegen bei $80 \%$ [2].

Die LVAD werden v.a. elektiv und nach präoperativer Konditionierung operativ implantiert, wenn eine langfristige Unterstützung indiziert ist. Durch eine grundlegend geänderte ventrikuläre Hämodynamik nach Implantation des LVAD - v.a. mit kontinuierlichem Blutfluss - kann es u. U. intra- oder postoperativ zum Rechtsherzversagen kommen. Dies ist meist bedingt durch eine nicht an die linksventrikuläre Entlastung adaptierte v.a. septale myokardiale Dysfunktion. Daher empfehlen sich zunächst die detaillierte präoperative Abklärung sowie eine engmaschige Überprüfung der RV-Funktion intra, peri- und postoperativ $[9,10]$.

Die Versorgung mittels RVAD ist selten erforderlich und erfolgt vorrangig als passagere Maßnahme bei rechtsventrikulärem Versagen, beispielsweise im Rahmen einer LVAD-Implantation. Nur wenige Systeme sind langfristig für den Einsatz als RVAD zugelassen, wenngleich sie technisch in der Lage sind, die rechtsventrikuläre Funktion komplett zu übernehmen und teilweise ausschließlich dafür entwickelt wurden.

Die Implantation eines BiVAD wird ausschließlich bei Patienten mit biventrikulärem Pumpversagen als mittelfristige Versorgung zur Überbrückung bis zu einer Transplantation verwendet: Entsprechend den internationalen und nationalen Registerdaten wird diese Operation in etwa $3 \%$ der Fälle durchgeführt (• Abb. 5; $[9,10]$.

Im Unterschied zu den VAD werden für die Einbringung des ,total artificial heart" (TAH) operationsbedingt beide Ventrikel reseziert. Diese Operation erfordert einen viel größeren Aufwand für Patienten und implantierende Chirurgen sowie betreuende Mediziner. 
L/RVAD Implantation

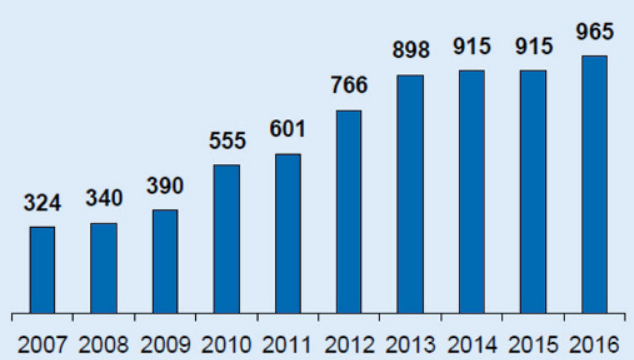

TAH

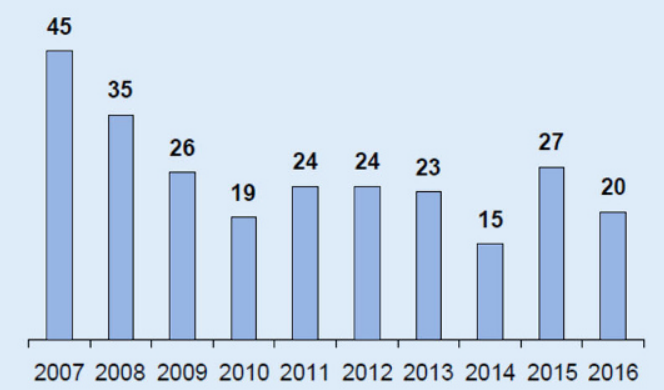

2007200820092010201120122013201420152016

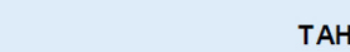

BVAD

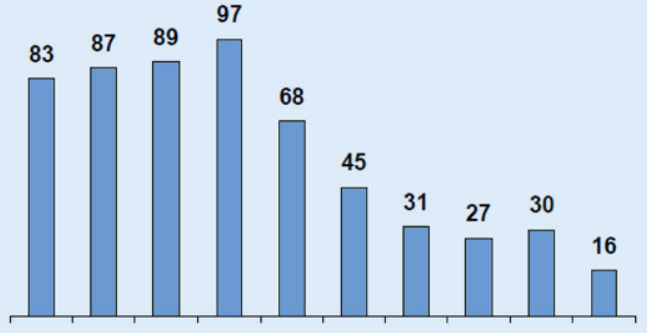

2007200820092010201120122013201420152016
Allen Geräten ist gemein, dass sie ein striktes Antikoagulationsregime erfordern, gegenwärtig v. a. aus Marcumar und einem Thrombozytenaggregationshemmer in Kombination bestehend, um thrombembolische Komplikationen zu verhindern. Dies birgt andererseits die Gefahr von Blutungen in sich. Darüber hinaus besteht die Komplikationsgefahr einer Infektion, v. a. durch die sog. Driveline $[9,10]$.

\section{Postoperatives Prozedere}

Die Pulslosigkeit und der u. U. nichtmessbare Blutdruck stellen eine Herausforderung für Ärzte und Patienten dar. Sie erfordern eine Umstellung der gewohnten Behandlungsmodalitäten für diese Patienten, insbesondere, wenn medizinische oder chirurgische Maßnahmen erforderlich sind, die mit der kardialen Situation nicht direkt im Zusammenhang stehen. Daher empfiehlt sich die Kontaktaufnahme mit dem implantierenden Zentrum.

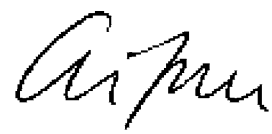

Sandra Eifert

\section{Korrespondenzadresse}

\section{Prof. Dr. S. Eifert}

Herzchirurgische Klinik und Poliklinik, Transplantationszentrum, Klinikum Großhadern, LMU München

Marchioninistr. 15, 81377 München,

Deutschland

Sandra.Eifert@med.uni-muenchen.de

Interessenkonflikt. S. Eifert gibt an, dass kein Interessenkonflikt besteht.

\section{Literatur}

1. RoseEA,Gelijns CA, MoskowitzAJetal(2001) Longterm use of a left ventricular assist device for endstage heart failure. N Engl J Med 345:1435-1443

2. Strüber M, Meyer AL, Malehsa D, Kugler C, Simon AR, Haverich A (2009) Situation der Herztransplantation und Weiterentwicklung von Kunstherzen. Dtsch Arztebl Int 106(28-29):471-477

3. Beyersdorf F, Martin J, Zehender $M$ et al (2005) Chirurgische Behandlungsoptionen bei terminaler chronischer Herzinsuffizienz. Dtsch Arztebl 102(37):A-2468 (B-2076/C-1966)

4. Moazami N, Fukamachi K, Kobayashi M et al (2013) Axial and centrifugal continuous-flow rotary pumps: a translation from pump mechanics to clinical practice. J Heart Lung Transplant 32(1):1-11

5. Mehra MR, Goldstein DJ, Uriel N et al (2018) Two-year outcomes with a magnetically levitated cardiac pump in heart failure. MOMENTUM 3 investigators. NEngl J Med 378(15):1386-1395

6. Derwall M, Moza A, Brücken A (2018) Die mechanische Kreislaufunterstützung in der
Abb. $5<$ DGTHG-Statistik: Implantierte VAD-Systeme. LVAD linksventrikulär, RVAD rechtsventrikulär, BiVAD biventrikulär, $T A H$ total artificial heart

Sandra Eifert

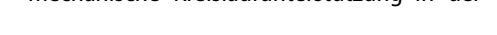

